Canu, W. H., \& Carlson, C. L. (2007). Rejection sensitivity and social outcomes of young adult men with ADHD. Journal of Attention Disorders, 10(3): 261-275 (Feb 2007). Published by SAGE (ISSN: 1557-1246). doi:10.1177/1087054706288106

\title{
Rejection Sensitivity and Social Outcomes of Young Adult Men with ADHD
}

Will H. Canu and Caryn L. Carlson

\begin{abstract}
Objective: Attention-Deficit/Hyperactivity Disorder (ADHD) has been consistently linked to social maladjustment. This study investigated whether elevated rejection sensitivity (RS) could contribute to the relational problems that adults with ADHD encounter. Method: Undergraduate men in ADHD-Combined Type (ADHD-C; $n=31$ ), ADHD-Primarily Inattentive Type (ADHD-IA; $n$ $=22$ ), and nondiagnosed control (NC; $n=25$ ) groups completed questionnaires concerning RS, relational history, current relationships, and self-esteem. Results: The hypothesis that those with ADHD would have elevated RS (versus NC peers) was not supported. However, low RS predicted divergent outcomes across groups. Furthermore, ADHD-IA men reported more negative relational outcomes than their ADHD-C peers, although both groups reported lower general self-esteem than controls. Conclusion: Perhaps the positive illusory bias associated with childhood ADHD could buffer RS development. Findings provide evidence of ADHD-related impairment in adult relationships, further differentiate the principal ADHD subtypes, and extend the RS literature to this clinical population.
\end{abstract}




\section{INTRODUCTION}

Empirical studies have consistently indicated that impaired social functioning is a common outcome for many children with Attention-Deficit/Hyperactivity Disorder (ADHD; Gaub \& Carlson, 1997; Hoza et al., 2005; Landau \& Moore, 1991). These children are judged and rejected quickly (Milich \& Landau, 1982; Pelham \& Bender, 1982), receiving little sympathy even from ADHD-mates (Erhardt \& Hinshaw, 1994). Aggression, labile emotions, and overly abrupt verbalizations-more often associated with hyperactivity-impulsivity $(\mathrm{HI})$ than inattention (Henker \& Whalen, 1999; Maedgen \& Carlson, 2000)—have been cited as important factors in the maladaptive social interactions of boys with ADHD (e.g., Hinshaw \& Melnick, 1995). Furthermore, children with ADHD_particularly those with prominent $\mathrm{HI}$ symptoms-are also likely to suffer disrupted relationships within their families (Edwards, Barkley, Laneri, Fletcher, \& Metevia, 2001; Johnston, Murray, Hinshaw, Pelham, \& Hoza, 2002). Although substantially fewer empirical studies have focused on adolescents and adults with ADHD, a growing body of work suggests that difficulties in peer relationshipsfriendships (Weiss, Hechtman, Perlman, Hopkins, \& Wener, 1979) and romances (Canu \& Carlson, 2003; Murphy \& Barkley, 1996; Weiss, Hechtman, Milroy, \& Perlman, 1985)—persist in later life.

Given that prior research has shown $\mathrm{HI}$ symptoms to decrease over the lifespan (Biederman, Faraone, \& Mick, 2000), social adjustment in late adolescence and adulthood may actually improve, at least for those with the ADHDCombined Type (ADHD-C), given the negative influence that $\mathrm{HI}$ symptoms and related behaviors (e.g., aggression) have on peer relationships. However, this has not consistently been shown to be the case in previous longitudinal studies (e.g., Weiss et al., 1985) in which ADHD-C was likely overrepresented (Wilens, Biederman, \& Spencer, 2002). Examining likely moderating variables could help explain the observed continuity of relational dysfunction. In addition, specifying ADHD-C and ADHD-Primarily Inattentive Type (ADHD-IA) is indicated to establish that social maladjustment occurs in both groups (Weiss, Hechtman, \& Weiss, 1999).

\section{Rejection Sensitivity and ADHD}

One cognitive-behavioral factor described in the social psychology literature that relates to social maladjustment is rejection sensitivity (RS), the tendency "to anxiously 
... expect, readily perceive, and overreact to rejection"

(Downey, Khouri, \& Feldman, 1997, p. 85). As

noted above, children with ADHD are likely to encounter

rejection at the hands of their peers and negative interactions

in their homes, as well. In fact, recent work has

linked childhood ADHD (Clarke, Ungerer, Chahoud, Johnson, \& Stiefel, 2002) and its frequently comorbid oppositional behavior (Gomez \& Gomez, 2002) to negative attachment, suggesting that even rejection by parents, in some cases, is chronic. Downey and colleagues propose that precisely these kinds of early rejection can lead to elevated RS, which in turn can influence relational adjustment through adulthood. Negative outcomes seen in children and adolescents with high RS include increased conflict with school peers, staff, and early dating partners, as well as lower teacher-rated social competence (Downey, Freitas, Michaelis, \& Khouri, 1998;

Purdie \& Downey, 2000). Adults with high levels of RS also exhibit jealousy and hostility in their romantic relationships, contributing to elevated partner dissatisfaction and predicting termination of relationships over a 1-year period (Downey \& Feldman, 1996; Downey et al., 1998).

There is continuity between RS and ADHD with regard to social engagement patterns. Adults with high RS often adopt one of two engagement styles in romantic relationships: high versus low relational investment (RI; Downey, Feldman, \& Ayduk, 2000). Those with high RI become socially preoccupied, exerting substantial energy to secure close relationships. Low RI, on the other hand, is associated with avoidance of any nonessential social interactions, which effectively reduces the overall chances of rejection and pain. Each of these coping strategies has been linked to negative social behaviors: physical aggression and social withdrawal, respectively (Downey et al., 2000). Similar behaviors have been noted in the social styles of children with ADHD. Henker and Whalen (1999) describe an "active/maladroit" social type associated primarily with ADHD-C characterized by an overly intense drive for interaction that irritates peers, and a "reluctant/avoidant" type that tends to observe rather than engage in social situations and is most common in children with ADHD-IA. Could this be early evidence that children with ADHD can develop higher RS than peers, adopting coping styles that "fit" according to subtype? 


\section{A Competing Perspective: Positive Illusory Bias}

Given that chronic rejection in childhood often co-occurs with ADHD, it seems possible that RS contributes to their enduring social problems. However, researchers have documented a positive illusory bias, in which children with ADHD make more external causal attributions for social and other failures than nondiagnosed peers (e.g., Diener \& Milich, 1997; Hoza, Waschbusch, Pelham, Molina, \& Milich, 2000). Although such causal attributions are distorted, simple perception of negative (e.g., awkward, upsetting) events does not appear to be impaired (Landau \& Moore, 1991; Ohan \& Johnston, 2002). An important issue to resolve, then, is whether perception of social rejection alone could cause ADHD children to develop higher RS or whether the overly positive causal attributions of such children could inhibit such an outcome.

\section{Aims of This Study}

This study follows up a prior investigation of the romantic relational outcomes of undergraduate men with elevated symptoms of ADHD (Canu \& Carlson, 2003). This prior work suggested that men with ADHD-IA tended to be less assertive, less experienced, and more negatively evaluated by female confederates, as compared with those with ADHD-C and nondiagnosed peers. This study extends the previous one by examining $\mathrm{RS}$, a factor that may moderate the long-term social outcome of men with ADHD.1 The primary aim is to evaluate whether level of RS differentiates the major subtypes of ADHD from nondiagnosed peers and to document whether low RS serves as a buffer against negative relational outcomes in ADHD groups. In addition, group outcomes on romantic (e.g., current satisfaction, past sexual and dating experience), friendship (e.g., perceived support), and self-relational (i.e., selfesteem) adjustment are also examined.

\section{METHODS}

\section{Participants}

Male, heterosexual participants between the ages of

18 and 24 were recruited in 2003 from a large public university $(75 \%)$ and an urban community college $(25 \%)$ in the Southwestern United States using advertisements posted by the authors (e.g., at public kiosks) and announcements from staff members at these institutions (e.g., e-mail 
from Office for Students with Disabilities). Comparison groups were nondiagnosed control (NC) participants $(n=25)$ and those with ADHD-C $(n=31)$ or ADHD-IA $(n=22)$. A majority were Caucasian $(60 \%)$, although Asian (23\%), Hispanic (9\%), and multiethnic (8\%) backgrounds were represented. The average family-indexed socioeconomic status (SES; i.e., highest-rated parent vocation) was in the professional range. A more detailed demographic and psychiatric description of the sample is included in Table 1.

A diagnostic telephone screening (administered by the first author; Barkley \& Murphy, 1998) largely focusing on Diagnostic and Statistical Manual of Mental Disorders (4th ed.; DSM-IV; American Psychiatric Association, 1994) ADHD diagnostic criteria facilitated group assignment of the 113 men who responded. Inclusion criteria for those in the ADHD group were self-report of sufficient symptoms of $\mathrm{ADHD}(=6 \mathrm{HI}$ and $=6 \mathrm{IA}$ symptoms for ADHD-C, = 6 IA symptoms for ADHD-IA) exhibited beginning in childhood (younger than age 12; for rationale, see Barkley \& Biederman, 1997), impairment due to these symptoms in multiple domains, a prior diagnosis of ADHD by a mental or medical health professional, current enrollment at one of the institutions, and age between 18 and 24 years. Twenty-two responding to recruiting aimed at men with ADHD failed to meet inclusion criteria; another 13 were unable to participate due to logistical problems (e.g., disconnected phone, failed to appear). All those included in the NC group reported no diagnosis or significant symptoms of ADHD and had scores on diagnostic questionnaires that were within normal limits. One NC participant was excluded from analyses due to elevated $(>+1 S D$ above normed mean) scores on Wender Utah Rating and Conners Adult ADHD Rating scales administered during in-person data collection (see below).

Participants were paid $\$ 20$ for completion of study procedures. Those who were currently in romantic relationships $(n=30)$ were asked to give a confidential questionnaire packet to their partner; in addition to forms (e.g., cover letter, relational satisfaction survey; see below), a $\$ 2$ payment and a stamped, return envelope were included. The overall response rate for these romantic partners was $40 \%(n=12)$. The romantically involved participants reported their current relationships to have a mean duration of 15 months $(S D=11)$. 


\section{Measures}

Telephone screening interview. A structured, brief (30- to 45-minute) interview conducted by the first author, largely reviewing ADHD, ODD, and CD symptoms and modeled after the procedures of Barkley and Murphy (1998), assessed DSM-IV diagnostic status (e.g., symptom presence, impairment, age of onset) for childhood (i.e., ages 5-12) and current timeframes. The +1.5 SD threshold (i.e., nominations of often or very often) for retrospectively recalled childhood symptoms in the IA cluster is 7.5, and 7.2 in the $\mathrm{HI}$ cluster $(N=175)$. Supplemental questions gathered demographic information relevant to study participation (e.g., age, student status, sexual preference, previous diagnosis of ADHD, profession of diagnosis provider).

Conners Adult ADHD Rating Scale-Self Report: Screening Version (CAARS-S:SV). The CAARS-S:SV (hereafter shortened to CAARS; Conners, Erhardt, \& Sparrow, 1999) is a 30-item questionnaire designed to probe current manifestations of ADHD in adults. Eighteen items provided two factor scales used in this study: DSM- IV Inattentive Symptoms and DSM-IV Hyperactive-Impulsive Symptoms (.81 and .64 1-month test-retest reliabilities, respectively). The Inattentive and Hyperactive-Impulsive scales have high positive correlations with matching DSM-IV symptom clusters (.89 and .74 , respectively) in males (Conners et al., 1999).

Wender Utah Rating Scale (WURS). This abbreviated form of the WURS (Ward,Wender, \& Reimherr, 1993) is a self-report made up of 25 items shown to discriminate ADHD and NC samples. Responses are on a 5-point scale ( $0=$ not at all or very slightly, $4=$ very much). A total score of 36 yields $96 \%$ sensitivity and specificity for ADHD. The reported Spearman-Brown split-half $r=.9$ (in non-ADHD adults, $N=100$ ); Pearson correlations with Parent Rating Scale (Conners, 1973) scores were .49 for nondiagnosed controls and .41 for adults with ADHD, suggesting adequate concurrent validity, particularly as both measures were retrospective and completed by different informants.

Brief cognitive screening. Two subtests-Information and Picture Completion-from the Wechsler Adult Intelligence Scale-Revised (WAIS-R; Wechsler, 1981) were administered to participants by the first author. This brief form is considered to produce a valid estimate of Full Scale IQ (FSIQ; $r=.88$ for the norming sample). Estimated FSIQ was derived using Kaufman's (1990) formula for this dyad of scores. 
Beck Anxiety Inventory (BAI) and Beck Depression Inventory (BDI). The BAl (Beck \& Steer, 1990) is a 21-item measure of somatic and cognitive aspects of anxiety. Participants rate how much each symptom has bothered them in the past week on a 4-point scale $(0=$ not at all, 3 = severely). The BDI (Beck, 1967) assesses the presence and severity of depression. Participants select statements corresponding to a 4-point scale of depressive severity in the past 2 weeks. The abbreviated $\mathrm{BDI}$ (13 items) was used for this study ( $r=.96$ with original version; Beck \& Beck, 1972). Both measures have shown good split-half and test-retest reliability, and concurrent validity (Beck \& Beck, 1972; Beck \& Steer, 1990).

Rejection Sensitivity Questionnaire (RSQ). The RSQ (Downey \& Feldman, 1996) is a 36-item self-report measure of anxious expectations of rejection on 18 social situations relevant to adult interactions (e.g., "You go to a party and notice someone on the other side of the room, and then you ask them to dance"). Participants indicate the level of anxiety they have about each situation on a 6-point scale (1 $=$ very unconcerned, $6=$ very concerned). Participants then report expectancy that the other person (or persons) would respond in an accepting way ( 1 = very unlikely, 6 = very likely). The RS score for each situation is calculated by multiplying the expectancy of rejection $(=7-$ expectancy of acceptance) by the associated degree of anxiety. The overall RS scale score is the mean of the 18 situational RS scores. The RSQ has a high reported internal $(\alpha=.83)$ and 4-month test-retest reliability $(\alpha=.78)$ and has been shown to predict perceived rejection, negative attributions toward romantic partners, and relational insecurity.

Dating and sexual inventory. This questionnaire inquires about the breadth and timing of romantic experience. Fourteen items tap the age that dating (e.g., first dating attempt, first steady dating; $n=4$ ) and sexual (e.g., manual genital stimulation, vaginal intercourse; $n=10$ ) milestones were reached. Sexual milestone items were expanded from those in the National Survey of Adolescent Males (Gates \& Sonestein, 2000). While presented on a scale, responses were the whole-numbered ages corresponding to timing of first experience (or an indication that the milestone had not yet been reached). One item asked participants to list, by initials, all of their romantic partners and the length, in months, of the relationship $(<1$ month $=1$ ). Three questions addressed how many women the participant has attempted to date in the past year, how many accepted, and if the participant would like to date more frequently. As appropriate, participants also completed 
questions concerning current romantic relationships (e.g., frequency of dates with partner).

Investment in romantic relationships index (Investment index). This is a 2-item index previously used by Downey and colleagues (2000). Participants responded to two statements attributing importance to establishing a romantic relationship on a 4-point scale ( $1=$ not really true of me, 4 = really true of me). The reported Pearson correlation between the items is .77, and this index is significantly correlated with avoidant attachment $(r=-.22)$.

Locke-Wallace Marital Adjustment Test (LWMAT). The wording of this 15-item self-report measure (Locke \& Wallace, 1959) was modified slightly to pertain to the typical romantic relationships of this sample and taps relational satisfaction. Items are scaled and weighted differently; the range of adjustment scores on the LWMAT is 2 (extremely negative) to 158 (extremely positive). Scores below 100 are in a maladjusted range (Corcoran $\&$ Fischer, 1987). Internal reliability is reported as very good (Spearman-Brown $r=.90$ ).

Friendships questionnaire. This is a 10 -item measure that examines breadth and perceived support of friendships. Participants reported initials of "close friends" (not including romantic partner or close family) and frequency of contact as rated on a 7 -point scale $(1=$ once a month or less, $4=1-2$ times per week, $7=$ once or more every day). Overall perceived support from this network of friends was assessed using the 9-item friend support scale of the Provision of Social Relationships measure (Turner, Frankel, \& Levin, 1983). An example item is, "When I'm with my friends, I feel completely able to relax and be myself." Responses are made on a 5-point scale $(1=$ very much like me, $5=$ not at all like me). Satisfactory internal reliability has been reported $(\alpha=.75$ to .87), as well as a negative association with psychological distress (Brief Symptom Inventory score; $r=-.40$ ).

Rosenberg Self-Esteem Questionnaire (SEQ). The SEQ (Rosenberg, 1979) is a 10-item scale assessing selfimage; a short form of 8 items that maximize internal reliability ( $\alpha=.84$; Ayduk, Mendoza-Denton, Mischel, \& Downey, 2000) was administered and scored using Rosenberg's original instructions. The SEQ has a reported 2-week test-retest reliability of .85 . 


\section{Procedure}

Upon successful completion of a telephone screening interview, participants were individually scheduled for data collection sessions. To ensure accuracy of results on the cognitive screening measures, participants in the ADHD groups who were currently taking stimulant medication $(49 \%)$ were instructed to refrain from taking this medicine on the day of data collection. Procedures were completed in a private laboratory room, with individual sessions lasting 11/2 hours (including an experimenter-participant interaction not discussed here); the first author personally administered all measures. After written consent was obtained, the cognitive screening tasks were conducted. Participants then completed all questionnaire measures described here (with the exception of romantic partner RSQ and LWMAT forms, which were completed and returned independently); forms were given in a standardized order and labeled with only a participant number. To maximize confidentiality, when questionnaires were complete, participants placed them in an unmarked manila envelope, sealed it, and then placed it in a drop box, from which it was later collected. Cash payments (\$20.00) were made at the end of sessions; all participants were also given a list of community mental health and academic support services for future self-referral.

\section{Data Analytic Strategy}

Analysis of variance (ANOVA) procedures were used to assess for group differences on demographic, diagnostic, and dependent variable measures. Where variables were expected to vary together and were conceptually tied, multivariate ANOVA (MANOVA) was used to reduce Type I error. Two-tailed $t$ tests were employed for pairwise comparisons. In cases where a violation of homogeneity of variance assumptions was detected, nonparametric Kruskal-Wallis tests-which make no homogeneity of variance assumption-were employed. When a KruskalWallis chi-square $\left(X^{2}\right)$ was significant, nonparametric Mann Whitney tests (producing the $U$ statistic) were used in lieu of $t$ tests. Effect sizes (Cohen's $d$ ) were calculated to illustrate the magnitude of select group differences. Five sets of three multiple regression analyses were planned to examine the effect of RS, group assignment, and any interaction thereof on dating milestones composite score, 2 sexual milestones composite score,3 percentage of time in relationships since dating began, number of women dating was attempted with in past year, and ratio of success to failure in those attempts. One analysis was conducted for 
each pairwise combination (NC/ADHD-C, NC/ADHD-IA, ADHD-C/ADHD-IA), with RS, group assignment, and the interaction term (RS $\times$ group) entered simultaneously in one block of predictor variables. Finally, chi-square analyses were planned to examine whether "high" or "low" RS related to two outcomes in the ADHD groups: "Are you currently in a romantic (dating or more serious) relationship?" and "Would you like to date more frequently?" Calculations outlined by Cohen (1992) suggested that the planned statistical comparisons, setting the power at .80 and using the standard alpha level $(\alpha=.05)$, would generally detect medium-to-large differences in this sample.

\section{RESULTS}

\section{Demographic Variables}

Mean scores and standard deviations for demographic and descriptive variables are provided in Table 1.

ANOVAs were conducted to examine group differences on estimated FSIQ, age, and education level. None of these indicated significant differences [estimated FSIQ $F(2,74)=.14$, age $F(2,74)=.34$, education level $F(2,74)$ $=2.37$, all $n s]$. A Kruskal-Wallis test was used to examine group differences for socioeconomic status (SES: higher of parents' vocations; Stevens \& Featherman, 1981). The results of this test were also nonsignificant $\left[X^{2}(2, N=77)\right.$ $=1.33, n s]$. Given the overall lack of notable group differences, further analyses were not controlled for variance on these demographic variables. 
Table 1

Demographic and Descriptive

Characteristics of Sample

\begin{tabular}{lccc}
\hline & $\begin{array}{c}\text { NC } \\
(n=24)\end{array}$ & $\begin{array}{c}\text { ADHD-C } \\
(n=31)\end{array}$ & $\begin{array}{c}\text { ADHD-IA } \\
(n=22)\end{array}$ \\
\hline Age (years) & $20.8(1.4)$ & $21(1.9)$ & $21.1(1.3)$ \\
SES (family) & $60.3(21.8)$ & $67.7(17.8)$ & $66.6(12.8)$ \\
Education & $2.0(1.4)$ & $1.8(1.3)$ & $2.6(1.2)$ \\
FSIQ estimate & $104.2(10.8)$ & $105.5(11.6)$ & $105.6(8.3)$ \\
Childhood ADHD-IA & $1.1(1.7)^{\mathrm{a}}$ & $8(1.1)^{\mathrm{b}}$ & $7.6(1.2)^{\mathrm{b}}$ \\
$\quad$ symptoms & & & \\
$\quad$ HI symptoms & $1.3(1.4)^{\mathrm{a}}$ & $7.9(1.1)^{\mathrm{c}}$ & $3.7(1.4)^{\mathrm{b}}$ \\
$\quad$ Impaired domains & - & $3.8(1)$ & $3.3(1.2)$ \\
$\quad$ Est. onset (years) & - & $6.7(1.9)$ & $8.1(2.3)$ \\
Adulthood ADHD-IA & $0.7(1.1)^{\mathrm{a}}$ & $6.9(1.8)^{\mathrm{b}}$ & $6.8(1.9)^{\mathrm{b}}$ \\
$\quad$ symptoms & $1.0(1.3)^{\mathrm{a}}$ & $6.2(2.1)^{\mathrm{c}}$ & $3.4(1.8)^{\mathrm{b}}$ \\
$\quad$ HI symptoms & - & $4.5(2.4)$ & $4.5(1.9)$ \\
$\quad$ Impaired domains & $-9(9.5)^{\mathrm{a}}$ & $79.4(11.3)^{\mathrm{b}}$ & $79.9(8.7)^{\mathrm{b}}$ \\
CAARS-IA $t$-score & $48.9\left(1.5(8.2)^{\mathrm{a}}\right.$ & $72.6(12.8)^{\mathrm{c}}$ & $57.5(11.7)^{\mathrm{b}}$ \\
CAARS-HI $t$-score & $46.5 .5(9.3)^{\mathrm{a}}$ & $52.9(14.9)^{\mathrm{c}}$ & $41(11.6)^{\mathrm{b}}$ \\
WURS & $13.5 .1(3)^{\mathrm{a}}$ & $6.7(5.2)^{\mathrm{b}}$ & $8.6(7.6)^{\mathrm{b}}$ \\
BDI & $4.8(4.8)^{\mathrm{a}}$ & $12.8(9.7)^{\mathrm{b}}$ & $10.3(10.3)^{\mathrm{b}}$ \\
BAI & $4 \%(0 \%)$ & $31 \%(44 \%)$ & $9 \%(41 \%)$ \\
CD (ODD) history & &
\end{tabular}

Note: Superscript indicates pairwise differences of $p<.05$.

SES $=$ socioeconomic status (higher of parents' vocation; Stevens \& Featherman, 1981); FSIQ = Full Scale IQ; ADHD = Attention-

Deficit/Hyperactivity Disorder; IA = Inattentive; $\mathrm{C}=\mathrm{Combined}$; $\mathrm{NC}=$ nondiagnosed control $; \mathrm{HI}=$ Hyperactive $;$ CAARS $=$ Conners Adult ADHD Rating Scale; WURS = Wender Utah Rating Scale; $\mathrm{BDI}=$ Beck Depression Inventory; BAI = Beck Anxiety Inventory; $\mathrm{CD}=$ Conduct Disorder; ODD = Oppositional Defiant Disorder.

\section{Diagnostic Variables ${ }^{4}$}

A MANOVA was conducted to test for group differences on the following diagnostic variables: number of ADHD Inattention (IA) and Hyperactivity-Impulsivity (HI) symptoms reported for childhood and IA symptoms for adulthood (during telephone interview), WURS score, and $\mathrm{HI}$ and IA $t$-scores on the CAARS. As expected, the overall MANOVA test $[A=.04, F(12,138)=46.73, p<.01]$ and follow-up ANOVAs for these variables were significant [interview child IA symptoms $F(2,74)=216.54$, interview adult IA symptoms $F(2,74)=115.73$, interview child $\mathrm{HI}$ symptoms $F(2,74)=193.31$, CAARS IA $F(2,74)=$ 
77.10, CAARS HI $F(2,74)=37.40$, WURS $F(2,74)=$ 68.97; all $p s<.01]$. Pairwise two-tailed $t$ tests yielded an expected direction of differences (see Table 1). In addition, although well under the ADHD diagnostic cutoff for $\mathrm{HI}$ symptoms, the ADHD-IA group mean scores for $\mathrm{HI}$ measures were statistically higher than the control group's.

Kruskal-Wallis tests that examined differences on telephone interview adulthood $\mathrm{HI}$ symptom, BDI, and BAI reports were all statistically significant [interview adult $\mathrm{HI}$ symptoms $X^{2}(2, N=77)=46.73, p<.01$; BDI $X^{2}(2, N=77)=10.31, p<.01$; BAI $X^{2}(2, N=77)=12.77$, $p<.01]$. Follow-up Mann Whitney tests showed the same pattern noted for childhood HI symptoms as reported above as well as prototypically elevated depressive and anxious symptoms in the ADHD groups. As comorbidity is more the rule than the exception with the ADHD syndrome (see review in Pliszka, Carlson, \& Swanson, 1999) and a large majority of individuals in the ADHD groups did not exceed "borderline" elevations on either of the measures, BAI and BDI scores were not used as covariates in subsequent analyses.

Table 2

Group Means and Standard Deviations for Dependent Variables

\begin{tabular}{|c|c|c|c|}
\hline & $\mathrm{NC}(n=24)$ & ADHD-C $(n=31)$ & ADHD-IA $(n=22)$ \\
\hline RS self-report & $9.56(2.56)$ & $9.88(3.42)$ & $9.72(3.4)$ \\
\hline \# of women dated & $4.12(3.46)^{\mathrm{a}}$ & $8.74(6.03)^{\mathrm{b}}$ & $6.68(4.84)^{b}$ \\
\hline Time in relationships (\%) & $36.5(26.25)$ & $50.05(27.01)$ & $34.82(21.37)$ \\
\hline Average relationship length (months) & $7.8(8.82)$ & $4.81(3.66)$ & $4.63(4.18)$ \\
\hline \# of women asked to date in past year & $1.75(1.36)$ & $2.85(3.70)$ & $3.18(3.86)$ \\
\hline Dating initiation success, past year $(\%)$ & $84.13(32.69)$ & $89.96(26.49)$ & $88.04(18.95)$ \\
\hline Dating milestones composite & $5.31(4.08)$ & $4.39(2.55)$ & $4.58(1.6)$ \\
\hline Sexual milestones composite & $2.41(1.5)^{\mathrm{b}}$ & $1.84(.32)^{2}$ & $2.93(1.97)^{b}$ \\
\hline Amount of contact with close friends & $31.01(20.11)$ & $34.85(22.25)$ & $31.69(24.52)$ \\
\hline Friend support scale & $4.3(.58)$ & $4.17(.90)$ & $3.86(1.04)$ \\
\hline Self-esteem & $5(1.38)^{\mathrm{b}}$ & $3.52(1.77)^{\mathrm{a}}$ & $4.05(1.56)^{2}$ \\
\hline Investment index & $\begin{array}{c}6.42(1.79) \\
n=9\end{array}$ & $\begin{array}{c}6.87(1.63)^{b} \\
n=15\end{array}$ & $\begin{array}{c}5.91(1.38)^{2} \\
n=6\end{array}$ \\
\hline Self-rated LWMAT & $114.23(23.47)$ & $116.29(25.07)$ & $95.27(23.3)$ \\
\hline Frequency of dates in relationship & $3.89(1.17)$ & $4.27(1.71)$ & $3.17(1.72)$ \\
\hline Partner's LWMAT & $\begin{array}{c}n=4 \\
124.75(17.5)\end{array}$ & $\begin{array}{c}n=4 \\
131(17.32)\end{array}$ & $\begin{array}{c}n=4 \\
98.29(26.36)\end{array}$ \\
\hline
\end{tabular}

Note: Standard deviations are in parentheses. For dating and sexual milestones composites, lower scores equate to earlier and broader experience. For Investment index, ADHD-C group $n=30$ due to omission by one participant. Group scores followed by a superscript "a" were significantly $(p<.05)$ lower than those with a superscript " $b$ " for that same variable, upon pairwise analysis. ADHD =

Attention-Deficit/Hyperactivity Disorder; IA = Inattentive; $\mathrm{C}=$ Combined; $\mathrm{NC}=$ nondiagnosed control; RS = rejection sensitivity; LWMAT = Locke-Wallace Marital Adjustment Test (Locke \& Wallace, 1959).

Table 2 presents group mean and standard deviation values for all other dependent variables noted below. Group differences in RS. An ANOVA was conducted 
to examine differences on self-reported RS. Contrary to hypotheses, this analysis, as well as planned pairwise comparisons, showed no significant group differences $[F(2,74)=.07, n s]$. This finding runs counter to the hypothesis that nondiagnosed control participants and those in the ADHD groups would have diverging levels of RS.

Figure 1

Regression Lines for ADHD-Inattentive Type (-IA; $n=22)$ and ADHD-Combined Type $(-\mathrm{C} ; \boldsymbol{n}=\mathbf{3 0})$

Groups Depicting the Significant Interaction Effect of Self-Reported RS $\times$ ADHD Subtype on Dating Milestones Composite Score

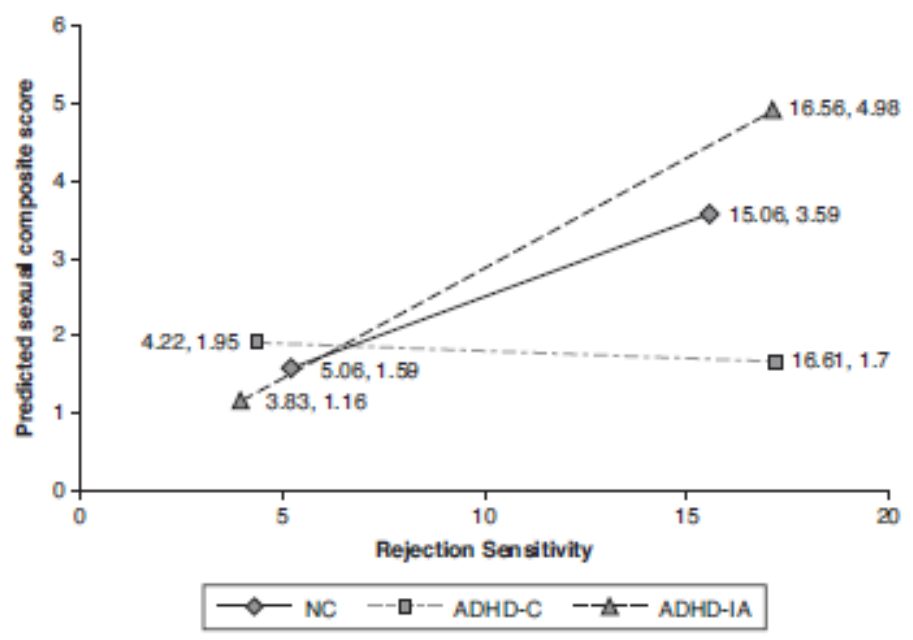

Note: Lower dating composite score means earlier and more extensive dating experience. The control group ( $\mathrm{NC} ; n=23$ ) regression line is also plotted for visual comparison. Lines shown here represent the entire range of response within these groups for RS. Point values listed are for X (RS) and Y (predicted dating milestones composite score) axes. 


\section{Figure 2}

Regression Lines for Nondiagnosed Controls (NC; $n=21)$ and ADHD-Combined Type $(-\mathrm{C} ; n=28)$

Groups Depicting the Significant Interaction Effect of Self-Reported Rejection Sensitivity (RS) $\times$ Group on Success in Dating Attempts in the Past Year

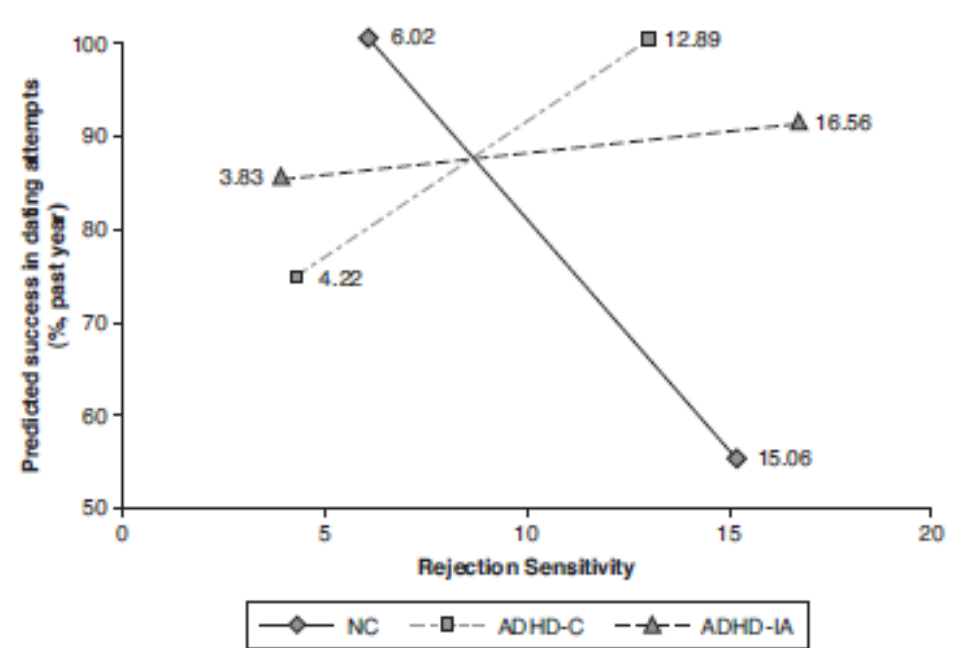

Note: These lines are capped where the predicted value of success in dating attempts reaches $100 \%$; the actual range of RS for the NC group is 5.06 to 15.06, and for the ADHD-C group is 4.22 to 16.56. Point values listed are for the $\mathrm{X}$ axis (RS). The ADHD-Inattentive type group $(-\mathrm{IA} ; n=22)$ regression line is also plotted for visual comparison and shows the entire range of predicted success for this group. 
Figure 3

Regression Lines Depicting Rejection Sensitivity (RS) and Predicted Sexual Milestones Composite Score in the Nondiagnosed Control (NC; $n=21$ ),

ADHD-Inattentive Type (-IA; $n=22)$, and

ADHD-Combined Type ( $-\mathrm{C} ; n=29)$ Groups

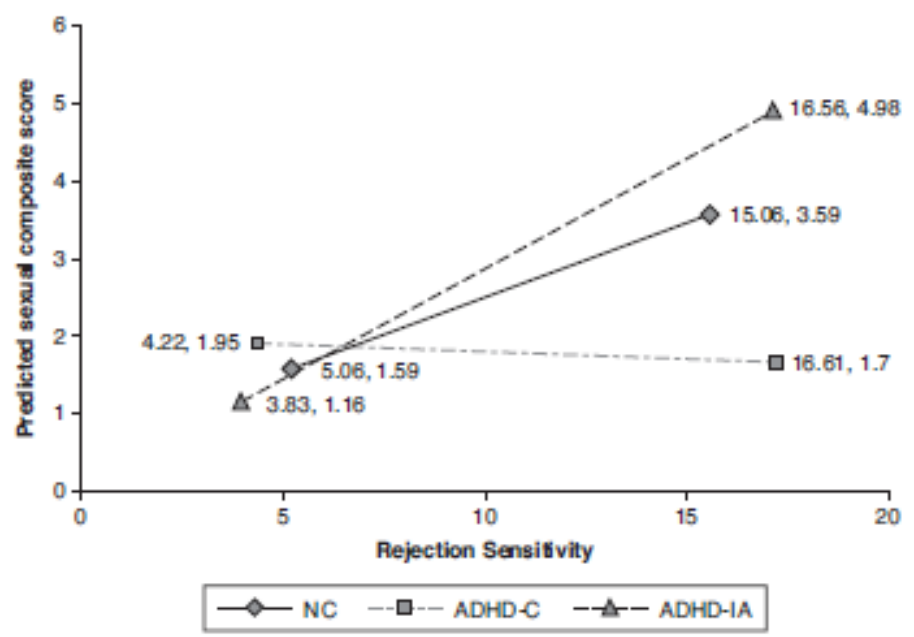

Note: Significant interaction effects were noted for RS and group in multiple regressions for $\mathrm{NC}$ and $\mathrm{ADHD}-\mathrm{C}$ as well as ADHD-IA and ADHD-C combinations. As noted in Table 2, lower sexual composite scores mean earlier and broader sexual experiences. Lines shown here represent the entire range of response within these groups for RS. Point values listed are for X (RS) and Y (predicted sexual milestones composite score) axes.

$R S$ buffering effects: Multiple regressions. The pattern of results on the multiple regression analyses indicated diverging effects of RS between groups. Partially supporting our hypothesis and keeping with previous RS research in the general population, low RS among NC and participants with ADHD-IA predicted more "positive" 5 relational outcomes on two out of five variables for each group (NC: earlier and diverse sexual milestones and higher ratio of dating initiation success in past year; ADHD-IA: earlier and diverse sexual milestones and dating milestones), suggesting a selective buffering effect. In surprising contrast, high RS predicted a better outcome in the ADHD-C group on two out of five variables (earlier and diverse dating milestones and higher initiation success in past year), and low RS did not predict any "positive" relational outcomes. Figures 1 
through 3 depict the interaction effects detected in these analyses; for simplicity of reporting here, a complete summary of the derived regression statistics is noted in Table 3.

Table 3

Rejection Sensitivity Multiple Regression Analyses Results

\begin{tabular}{|c|c|c|c|}
\hline Variable & Groups & Multiple Regression & Significant Predictors \\
\hline \multirow{5}{*}{ Dating milestones composite } & 1,2 & $R=.25, F(3,49)=1.12, n s$ & - \\
\hline & 1,3 & $R=.28, F(3,41)=1.18, n s$ & - \\
\hline & 3,2 & $R=.4, F(3,48)=3.04, p=.04$ & $\mathrm{RS}: \beta=.45, p=.03 ; b=.29$ \\
\hline & & & Group: $\beta=1.12, p=.01 ; b=4.88$ \\
\hline & & & Interaction: $\beta=-1.31, p<.01, b=-.52$ \\
\hline \multirow[t]{3}{*}{ Time in relationships $(\%)$} & 1,2 & $R=.29, F(3,51)=1.55, n s$ & - \\
\hline & 1,3 & $R=.26, F(3,42)=.99, n s$ & - \\
\hline & 3,2 & $R=.35, F(3,49)=2.28, n s$ & - \\
\hline \multirow[t]{3}{*}{ Number of women asked on dates in past year } & 1,2 & $R=.22, F(3,51)=.83, n s$ & - \\
\hline & 1,3 & $R=.31, F(3,42)=1.51, n s$ & - \\
\hline & 3,2 & $R=.14, F(3,49)=.31, n s$ & - \\
\hline \multirow[t]{3}{*}{ Ratio of success in dating attempts in past year } & 1,2 & $R=.38, F(3,45)=2.47, p=.07^{n s}$ & $\begin{array}{l}\text { Group: } \beta=-1.16, p=.03, b=-67.5 \\
\text { Interaction: } \beta=1.45, p=.01, b=7.87\end{array}$ \\
\hline & 1,3 & $R=.32, F(3,38)=1.47, n s$ & - \\
\hline & 3,2 & $R=.32, F(3,45)=1.7, n s$ & - \\
\hline \multirow[t]{3}{*}{ Sexual milestones composite } & 1,2 & $R=.41, F(3,46)=3.09, p=.04$ & $\begin{array}{l}\text { RS: } \beta=.58, p=.03, b=.20 \\
\text { Interaction: } \beta=-1.18, p=.04, b=-.22\end{array}$ \\
\hline & 1,3 & $R=.48, F(3,39)=3.8, p=.02$ & - \\
\hline & 3,2 & $R=.61, F(3,47)=9.18, p<.01$ & $\begin{array}{l}\text { RS: } \beta=.71, p<.01, b=.3 \\
\text { Group: } \beta=.72, p=.05, b=2.02 \\
\text { Interaction: } \beta=-1.26, p<.01, b=-.32\end{array}$ \\
\hline
\end{tabular}

Note: 1 = nondiagnosed control group; 2 = ADHD-Combined group; $3=$ ADHD-Primarily Inattentive group. ADHD = AttentionDeficit/Hyperactivity Disorder; $\beta=$ standardized beta weight; $b=$ unstandardized beta weight; RS = rejection sensitivity. Superscript " $n s$ ": Although this regression was nonsignificant, description of this analysis is included due to the significant interaction effect.

$R S$ buffering effects: Chi-square analyses. Using the sample mean for RS (9.73), members of the ADHD groups were assigned to either high RS (ADHD-C $n=15$, ADHD-IA $n=9$ ) or low RS (ADHD-C $n=16$, ADHDIA $n=13$ ) categories. Four chi-square analyses were used to evaluate possible differences between high- and low$\mathrm{RS}$ members within each ADHD group on the dichotomous items of "Are you currently in a romantic (dating or more serious) relationship?" and "Would you like to date more frequently?"

As noted in Table 4, only in the ADHD-IA group were the chi-square statistics significant. Phi correlations indicated a significant association between low RS and both being in a relationship and wanting to date more $(f=.51$, -.44 , respectively; both $p s<.05)$. Although these analyses do not have the predictive power of a regression, they suggest that, for the ADHD-IA group, low RS could play a buffering role with regard to these relational variables. 
Investment index. A two-tailed $t$ test was used to evaluate the hypothesis that ADHD-C and ADHD-IA groups would differ on romantic relational investment. As expected, individuals with ADHD-C reported higher investment index scores than those with ADHD-IA [t(50) $=2.23, p=.03$ ].

Table 4

Chi-Square Analyses on Dating Variables Between High and Low RS ADHD Subgroups

\begin{tabular}{lccccccc}
\hline & \multicolumn{3}{c}{ ADHD-C } & & \multicolumn{3}{c}{ ADHD-IA } \\
\cline { 2 - 3 } \cline { 6 - 7 } Variable & Low & High & & Low & High & \\
& RS & RS & $\chi 2$ & & RS & RS & $\chi 2$ \\
\hline Relationship? & & & & & & \\
No & 7 & 9 & & 7 & 9 & \\
& $(23 \%)$ & $(29 \%)$ & .82 & $(32 \%)$ & $(41 \%)$ & 5.71 \\
Yes & 9 & 6 & $n s$ & 6 & 0 & $p=.02$ \\
& $(29 \%)$ & $(19 \%)$ & & $(27 \%)$ & $(0 \%)$ & \\
Date more? & & & & & & \\
No & 6 & 5 & & 7 & 1 & \\
& $(20 \%)$ & $(17 \%)$ & .14 & $(32 \%)$ & $(5 \%)$ & 4.20 \\
Yes & 9 & 10 & $n s$ & 6 & 8 & $p=.04$ \\
& $(30 \%)$ & $(33 \%)$ & & $(27 \%)$ & $(36 \%)$ & \\
\hline
\end{tabular}

Note: $\mathrm{RS}=$ rejection sensitivity; $\mathrm{ADHD}=$ Attention-

Deficit/Hyperactivity Disorder; IA = Inattentive; $\mathrm{C}=$ Combined; Relationship? = "Are you currently in a romantic (dating or more serious) relationship?"; Date more? = "Would you like to date more frequently?" High and low RS were determined by assigning individuals based on the study sample mean (9.73). Numbers in parentheses $=$ percentage of overall ADHD group (e.g., ADHD-C or -IA). Total $N=30$ for ADHD-C group on "Date more?" because one participant omitted this response.

High $R S$ relational "buffer" in $A D H C$-C group.

Obviously, the trait that most distinguishes those with ADHD-C from the other participants is their prominent $\mathrm{HI}$ characteristics; could elevated RS somehow moderate negative outcomes from social behaviors that are specifically associated with $\mathrm{HI}$ ? Supplemental analyses were employed to evaluate whether men in the ADHD-C group with high $\mathrm{RS}$ reported more selective dating behaviors as compared with ADHD-C peers with low RS. Members of the ADHD-C group were again assigned to high $(n=15)$ and low $(n=16)$ RS conditions. A chi-square test was then used to determine whether 
these subgroups differed with regard to prevalence of high and low relational investment (split at the sample mean for investment index score $=6.45$ ). Although those in the ADHD-C/high RS subgroup more frequently reported high relational investment $(80 \%$, vs. $50 \%$ in the ADHD-C/low RS subgroup), this amounted to a nonsignificant trend $\left[X^{2}(1, N=31)=3.044, n s\right]$. Other possible indices (not already assessed in multiple regressions) of selectivity or restraint in dating initiation situations showed no significant differences between the ADHD-C/high-low RS subgroups: average length and lifetime number of romantic relationships [respectively: $t(29)=1.13, n s ; t(29)=.36, n s]$ and age at first date $[t(28)$ $=1.10, \mathrm{~ns}]$; whether "steady" dating has been accomplished $\left[X^{2}(1, N=31)=.301, n s\right]$. In short, the overall results of these analyses do not seem to support that those in the ADHD-C group with high RS date conservatively (as compared with those with ADHD-C/low RS).

\section{Romantic Relationships: Group Differences}

Duration and quantity. A Kruskal-Wallis test was used to examine average length of romantic relationship. Although the raw mean scores suggested that curtailed relationships were more common for those with ADHD, these differences were not significant $\left[X^{2}(2, N=77)=\right.$ $.65, n s]$, contradicting the initial hypothesis. A MANOVA examined two other variables: total number of women dated and the percentage of time in romantic relationships since dating began (see Table 2). This analysis yielded a significant overall result $[A=.83, F(4,146)=$ $3.67, p<.01]$. Subsequent analyses showed both ADHD groups to report a greater number of lifetime romantic relationships than controls $[\mathrm{F}(2,74)=5.75, p<.01$; ADHD-C vs. NC: $t(53)=3.57, p<.01$; ADHD-IA vs. NC: $t(44)=2.07, p=.04]$. Finally, although the ADHD-C group tended to report a higher percentage of time in romantic relationships (since the onset of dating), as compared with both other groups, these differences did not reach statistical significance $[F(2,74)=3.01, n s]$.

Current satisfaction. ANOVA analyses on participantand partner-reported LWMAT scores found no significant group differences in current relational satisfaction [respectively: $F(2,27)=1.69$, ns; $F(2,9)=2.58, n s]$, as did planned pairwise comparisons between the ADHD groups [respectively: $t(21)=1.77, n s$; partner $t(6)=2.07, n s$ ].

Achievement and timing of sexual milestones. A KruskalWallis test was employed to test for group differences on the 
sexual milestones composite score. The resulting $X 2$ and follow-up Mann Whitney comparisons showed ADHD-C participants to report earlier and more diverse sexual experience, as compared with both ADHD-IA and nondiagnosed peers $\left[X^{2}(2, N=72)=8.21, p=.02\right.$; ADHD-C vs. - IA $\mathrm{U}(50)=194.50, p=.02 ;$ ADHD-C vs. $\mathrm{NC} \mathrm{U}(49)=179.50$, $p=.01 ; \mathrm{ADHD}-\mathrm{IA}$ vs. $\mathrm{NC} U(42)=225.00, n s]$.

\section{Other Relational Constructs}

A MANOVA was executed to examine differences on self-esteem, the extent of contact with close friends day-today (see Table 2), and the friend support scale score. The overall result of the MANOVA was significant $[\mathrm{A}=.81$, $F(6,144)=2.68, p=.02]$.

Table 5

Selected Effect Sizes

\begin{tabular}{lccc}
\hline $\begin{array}{l}\text { dasdsa } \\
\text { asdsadas }\end{array}$ & $\begin{array}{c}\text { NC vs. } \\
\text { ADHD-C }\end{array}$ & $\begin{array}{c}\text { NC vs. } \\
\text { ADHD-IA }\end{array}$ & $\begin{array}{c}\text { ADHD-C vs. } \\
\text { ADHD-IA }\end{array}$ \\
\hline Investment index & -.26 & .32 & .60 \\
Self-rated LWMAT & -.09 & .77 & .81 \\
Frequency of dates in & -.25 & .51 & .63 \\
$\quad$ relationship & & & \\
\# of women dated & -.84 & -.59 & .37 \\
Time in relationship (\%) & -.5 & .07 & .59 \\
Sexual milestones composite & .55 & -.3 & -.77 \\
Friend support scale & .17 & .55 & .35 \\
Self-esteem & .84 & .62 & -.31 \\
\hline
\end{tabular}

Note: Effect size (absolute value) $>.20=$ small, $>.50=$ medium, $>.80=$ large (Cohen, 1992). ADHD = Attention-Deficit $/$ Hyperactivity Disorder; IA = Inattentive; $\mathrm{C}=$ Combined; $\mathrm{NC}=$ nondiagnosed control; LWMAT = Locke-Wallace Marital Adjustment Test (Locke \& Wallace, 1959). Negative effect sizes indicate lower values in the first group listed; positive values indicate higher values in the first group listed. Effect sizes are Cohen's $d$.

a. Lower scores equate to earlier and broader experience.

Friendships. Neither overall $[F(2,74)=1.86$, ns] nor planned pairwise $t$ tests revealed significant differences with regard to perceived support from friends, contrary to initial expectations. Similarly, there were no significant differences with regard to frequency of contact with close friends $[F(2,74)=.24, n s]$. 
Self-esteem. Results on the follow-up ANOVA for self-esteem $[F(2,74)=5.88, p<.01]$ and subsequent $t$ tests confirmed the a priori hypothesis that individuals in the nondiagnosed control group would have higher self-esteem than those in the ADHD-C $[t(53)=3.39$, $p<.01]$ and ADHD-IA $[t(44)=2.20, p=.03]$ groups.

Effect sizes. For brevity and convenience's sake, Table 5 consolidates effect size calculations, which demonstrate the magnitude of group differences for select variables.

\section{Medication Effects}

Three broad types of medication use were reported in the ADHD groups: none (36\%), stimulant (49\%), and nonstimulant (15\%). A MANOVA analysis on seven continuous dependent variables of interest-dating and sexual milestones composites, dating initiation success in the past year, relational investment index, percentage of time in romantic relationships, number of lifetime romantic partners, and self-esteem-returned nonsignificant results $[\mathrm{A}=.70, F(14,78)=1.04, n s]$, suggesting no notable medication effects on the findings reported here.

\section{DISCUSSION}

\section{Summary of Main Findings}

Contrary to our initial hypothesis, young men with ADHD did not report higher RS than their nondiagnosed peers. However, buffer effects of varying levels of RS on social outcomes appeared for the ADHD groups as well as their NC peers. Keeping with prior research on RS, the ADHD-IA and the NC groups had more positive outcomes associated with low $R S$. It is interesting that high $\mathrm{RS}$ predicted better social adjustment in the ADHD-C group. Although main effects across several social outcome variables were also examined, differences were relatively few and far between. The ADHD-C group reported earlier and more diverse sexual experience than others and also indicated higher investment in forming romantic relationships than their ADHD-IA peers. Further, both ADHD groups reported having more lifetime romantic partners than the NC group. Finally, both ADHD groups reported lower self-esteem than their NC counterparts, with the men with ADHD-C indicating the lowest self-regard. 


\section{General Discussion}

That these young men with ADHD did not have greater RS than their nondiagnosed peers was surprising given that many met criteria for comorbid disruptive behavior and also reported childhood impairment in family and/or peer relationships in the telephone interview (ADHD-C: 65\% and 68\%, respectively; ADHD-IA: $59 \%$ for both). How could a population that so commonly experiences chronic rejection in childhood not manifest higher RS? Perhaps this reflects an adulthood manifestation of the self-protective, "positive" illusory bias that has been documented in children with ADHD (Diener \& Milich, 1997; Hoza et al., 2000; Ohan \& Johnston, 2002) and is related to the overestimation of ability in performance (Milich \& Okazaki, 1991) and social tasks (Hoza, Pelham, Dobbs, Owens, \& Pillow, 2002).

This body of prior research suggests that children with ADHD who are rejected by their peers are unlikely to accurately perceive their high degree of impairment in peer relations. Extended developmentally, young adult men with ADHD may continue to underestimate the likelihood of rejection in social situations and, as a consequence, not feel anxious enough about others' reactions to register higher RS. Mrug and colleagues (Mrug, Hoza, \& Gerdes, 2001) point out that a social, self-protective bias in childhood may prove detrimental to acquisition of socially appropriate behavior. Although these findings can only support speculation, it seems possible that a benefit of this bias might be the prevention of elevated $\mathrm{RS}$, which in turn can serve a buffering role for negative adult relational outcomes. It may be fruitful for further research to more directly assess whether a positive, social illusory bias exists in adults with ADHD and, if documented, how it affects individuals at this later stage in life.

Some of the most interesting results stemmed from the hypothesis that low RS would play a buffering role against negative romantic outcomes, across groups. A series of studies has shown that high RS is socially disruptive in nonpsychiatric populations (Downey et al., 1998; Downey et al., 1997; Purdie \& Downey, 2000); this seems particularly true for adults in romantic relationships, where having low RS predicts more relational stability and success (Downey \& Feldman, 1996; Downey et al., 2000; Downey et al., 1998). Findings from this study provide some support for this effect; among the NC group, lower RS predicted a higher rate of successful dating initiation (in the past year) and earlier, more "serious" heterosexual experience. A similar buffer 
was suggested for those in the ADHD-IA group, as well, for whom low RS predicted early timing and variety of sexual and dating experience (and was also associated with being in a romantic relationship and with satisfaction concerning the amount of current dating). These results suggest that the phenomenon observed by Downey and colleagues can extend beyond the "normal" population to groups with psychological disorders.

A caveat to an overly broad generalization of this $R S$ buffering effect, of course, is the somewhat puzzling finding that low $\mathrm{RS}$ in the ADHD-C group predicted relatively negative romantic relational outcomes: later and less diverse dating experience and lower success at dating initiation. This suggests that this unique combination (high $\mathrm{HI}$ and low RS) is a liability for men. Downey and colleagues (1997) suggest that high RS individuals with elevated relational investment (as noted in the ADHD-C group, relative those with ADHD-IA) become preoccupied with securing "unconditional" romantic relationships and can be more selective, engaging only partners perceived as needy or very willing to commit in a relationship. Such a conservative dating strategy could represent a specific advantage for these men with ADHD-C in dating initiation contexts (i.e., an internal motivation to "put on the brakes"), where low RS counterparts may proposition women who are unlikely to accept (e.g., either of "too high" a standard or without high desire to jump headlong into a relationship) and suffer a higher rate of rejection. Analyses were conducted to see if a pattern of preferences and values could be detected to support this ad hoc hypothesis. A nonsignificant trend emerged for those with higher RS in the ADHD-C group to be more personally invested in having a romantic relationship (as compared with their ADHD-C/low RS peers), but there were no other indicators suggesting differences between these subgroups with regard to their romantic experiences.

Although these results did not support the ad hoc hypothesis, these relational outcome data (e.g., average length of relationship, number of partners, age at first date) do not conclusively disconfirm that those in the ADHDC/ high RS group may conservatively choose romantic partners, selectively targeting those they perceive as most likely to be receptive (e.g., commitment-oriented). Further research may replicate and then more effectively explore this curious effect of high RS in the ADHD-C group, perhaps by directly observing heterosocial behaviors (e.g., simulated or actual potential dating situation employing opposite-sex confederate), obtaining personality and/or 
dating preference data from partners (e.g., neuroticism, agreeableness, romantic relational investment), or using semistructured interviews or diary measures to specifically target behaviors and beliefs related to dating.

As expected, participants in the ADHD-IA group reported lower personal investment in romantic relationships as compared with their ADHD-C counterparts $(d=$ .60 ), indicating less drive to intimately connect with a female partner. This is consistent with observations made in children with ADHD-IA, in which they have been characterized as reluctant, shy, and standoffish in social situations. Findings from recent studies suggest that this "reluctant/avoidant" (Henker \& Whalen, 1999, p. 159) social style may, in fact, continue into adulthood. Canu and Carlson (2003) found that young men with predominant IA symptoms reported greater discomfort with situations demanding assertion, a later onset of dating, and were less interactive with and rated as less desirable partners by female confederates (as compared with peers with ADHD-C and those without ADHD). Other studies have also documented ongoing social skills deficits in samples of young adults with ADHD (not differentiated by type; e.g., Shaw-Zirt, Popali-Lehane, Chaplin, \& Bergman, 2005; Weiss \& Hechtman, 1993). Together, these findings provide some evidence of developmental consistency in social adjustment difficulties, particularly for those with ADHD-IA.

Some measures of heterosocial outcome did not clearly suggest that those in the ADHD groups differed from their nondiagnosed peers. In contrast to our a priori hypothesis, the mean duration of romantic relationship did not vary across groups. It appears, then, that when a romantic relationship is successfully initiated, those of men with ADHD endure about as long as most without ADHD. However, it should be noted that the mean length of relationship for the sample was less than 6 months $(M=5.69)$, which cannot be considered long-term for older individuals. Anecdotal accounts suggest that this may not be unusual; formal dating seems to be occurring less often in college populations, with temporary romantic pairings facilitated by parties or other group-oriented events becoming more common. Perhaps in an older sample (e.g., 28- to 35-year-olds) differences for mean relational duration between ADHD and nondiagnosed groups would emerge as the norm for romantic involvement shifts toward committed, longer term relationships. Research by Murphy and Barkley (1996) suggests just such a pattern for vocational maladjustment: By the mid-30s, when professional stability becomes more common, the number of 
instances of job loss (i.e., spontaneous quitting, being fired, or otherwise) in ADHD groups becomes statistically significant when compared with non-ADHD peers, whereas in younger samples, this relationship is not apparent (e.g., Hansen,Weiss, \& Last, 1999).

It is interesting that, relative to the NC group, a greater number of female lifetime dating partners was reported by both ADHD-C $(d=.84)$ and ADHD-IA $(d=.59)$

groups. This was expected for the ADHD-C group: Their potentially impulsive nature might lead to more frequent relationship initiation and dissolution, and previous research has indicated that low self-restraint and high misconduct (evidenced in this sample by prevalent ODD/CD comorbidity) in peer-rejected children are positively associated with number of sexual partners in adolescence (Feldman, Rosenthal, Brown, \& Canning, 1995). However, the ADHD-IA group result was unexpected, particularly given their lower relational investment and seemingly consistent developmental pattern of social withdrawal.

A count of participants reporting very few romances (i.e., 0 or 1 ) showed that this was more common in the NC $(n=7)$ than the ADHD-IA group $(n=2)$, and all but two of these "limited daters" (one from each group) reported a logical explanation (e.g., to concentrate on schoolwork, happy in their one and only relationship, personal convictions). This suggests that the difference between the control and ADHD-IA groups was driven by the prevalence of limited-yet-satisfied NC daters. In fact, when percentages of time in romantic relationships (since dating onset) were calculated and compared, the $\mathrm{NC}$ and ADHD-IA groups were equivalent, with the ADHD-C group tending to be higher than both $(d=.50$ and .59 , respectively), although even this difference was nonsignificant. Earlier and more diverse sexual experience was also reported by the ADHD-C group, relative to other groups ( $d=.55$ and .77 , respectively), consistent with previous research showing heightened sexual drive in young men with ADHD symptoms (Canu \& Carlson, 2003). In fact, a Pearson correlation showed that, for the complete sample, there was a positive association between time in romantic relationships (since dating onset) and early and diverse sexual experience (i.e., sexual milestones composite score; $r=.37 ; p<.01$ ).

Unfortunately, both the prevalence of current romantic involvement and the return rate of questionnaires from partners seriously diminished the statistical power to detect differences on the relational satisfaction measures. 
Although no significant differences were found, it is still interesting to note that the mean satisfaction of both

ADHD-IA participants and their partners was in the "dissatisfied" range ( $<100$ LWMAT score), whereas this was not the case for other groups. This result may relate to Robin and Payson's (2002) findings that, among married couples in which one partner has ADHD, six of the eight behaviors of the partner with ADHD that were most frequently nominated as eliciting perceptions of being unloved, unimportant, or ignored are IA characteristics (e.g., does not remember being told things, zones out in conversations).

Other results are consistent with the extant literature, including that adults with ADHD had lower general self-esteem than the NC group. Although the self-esteem finding is a replication of previous studies in both the child and adult ADHD literature, it is interesting to note that there was an even larger deficit noted for the ADHDC group $(d=.84)$ than the ADHD-IA group $(d=.62)$, despite the ADHD-C group's relative equivalence to NC participants on relational outcomes. This finding is all the more striking given that this sample of ADHD participants can by many indices be considered high functioning (e.g., average or higher IQs, attending college) and privileged (i.e., high SES). Further investigation is clearly needed to determine what factors in the cumulative experience of those with ADHD affect self-esteem.

\section{Limitations}

There are several limitations to this study that should be weighed when interpreting the findings. The sample size was small, limiting the power to detect group differences to medium-to-large effects. This shortcoming was problematic for some social adjustment variables (e.g., support derived from friendships, percentage of time in romantic relationships), but especially so for measures relating only to the subsample of current daters in the sample (e.g., self-rated LWMAT satisfaction) where group differences actually were medium-to-large in effect (see Table 5). Although we do not suggest that trends that did not reach statistical significance are robust evidence for social impairments in one or both ADHD groups, we hope that readers will regard these as "near misses" and indicative of areas that merit follow-up in larger samples.

ADHD status in this study was determined by self-reports of symptoms, impairment, and a previous diagnosis of ADHD obtained via a structured telephone 
interview. Further diagnostic information was gathered on self-report questionnaires. These criteria fall below a stringent standard that would be applied in many clinical settings; however, research has shown that ADHD selfreports have high discriminant validity (De Quiros \& Kinsbourne, 2001) and high concurrence with knowledgeable informant (e.g., parents, romantic partners) ratings

(Murphy \& Schachar, 2001). Within these parameters, however, participants included in the ADHD groups met the established DSM-IV criteria for symptom expression and impairment. A different criterion was used concerning age of onset; the DSM-IV specifies that symptoms must be present before age 7 , whereas this study used a standard of before age 12, a cutoff that others have shown to yield similar levels of impairment as the DSM-IV version (Barkley \& Biederman, 1997). Finally, comorbidity (i.e., ODD/CD, depression, anxiety) was neither an exclusion criteria nor a control variable in statistical analyses; it is so common in cases of ADHD that the ability to detect real-world differences between the ADHD subtypes and a nondiagnosed comparison group did not seem compromised.

This research exclusively sampled undergraduates, although perhaps a more diverse group than in previous studies. The high-functioning nature of the participants in this study is uncharacteristic of some with ADHD, and thus these results may not fully generalize to the entire ADHD population. One could speculate that academic achievement itself (inherent in our sample) could facilitate better social outcomes; this was not directly assessed but would not be expected given previously cited research concerning the acceptance of children with ADHD (Erhardt \& Hinshaw, 1994).

Finally, several measures of dating and sexual experience have been conceptualized in analyses and discussion as having straightforward positive or negative valences. However, early sexual experience, for example, is not an unambiguously positive outcome. Younger sexual partners may be more likely to have unprotected sex that could result in pregnancy or a sexually transmitted disease. Similarly, a higher rate of involvement in romantic relationships could be a sign of robust social skills and comfort, but could equally be born of dependence on having a partner to maintain self-esteem needs. The findings concerning adjustment in dating and sexual domains should be interpreted with this caveat in mind. 


\section{Implications}

Overall, it does not appear that RS distinguishes young adult males with ADHD from their nondiagnosed peers. It is suggested that the self-protective tendencies (i.e., reduced notice and incorporation of rejection and overestimation of social competency) that have been documented in prior research on childhood ADHD may influence the expression of RS in this population. Interesting differences were noted for the buffering effect of $\mathrm{RS}$ on romantic relational outcomes. Whereas the NC and ADHD-IA groups showed the expected pattern of low RS predicting better relational outcomes, the converse was detected for the ADHD-C group. The two ADHD groups were also distinguished by differences in romantic outcomes, suggesting that the childhood patterns of individuals with ADHD-C being more socially active and those with ADHD-IA being more withdrawn carry over into adulthood. Generally, adults with ADHDIA may be more at risk for negative social outcomes than their ADHD-C peers. As elsewhere, self-esteem was shown to be negatively associated with ADHD. This was notable given the high-functioning nature of this ADHD sample and was particularly striking for the seemingly socially well-adjusted ADHD-C group.

This study contributes to the body of knowledge concerning long-term social outcomes of individuals with ADHD. In addition, this work extends the literature on the buffering effects of RS to a clinical population, which, to the authors' knowledge, has not previously occurred. Given the divergent pattern of deficits shown by the ADHD-C and ADHD-IA groups, findings suggest that combining the ADHD types in adult samples assessing social outcomes is not warranted.

\section{Future Directions}

Several future directions are suggested. Observational data of ADHD participants interacting in a "friend" or "dating" role was lacking; further studies that collect such information would help to clarify whether the suppositions made from the data hold true. For example, the self-protective bias that has been noted in children is invoked here to explain certain results. However, it remains to be established experimentally whether this bias actually does carry over into adulthood. It would also be meaningful to explore how functional and dysfunctional relationships affect the behavioral and emotional adjustment of adults with ADHD. In addition, negligible 
data have been gathered to date pertaining to how others (i.e., romantic partners, friends, workmates) perceive adults with ADHD or how others' personalities may affect the outcome of relationships.

Clinical researchers have drawn substantially on biological, cognitive, developmental, and evolutionary psychology to better understand the ADHD phenomenon.

However, relatively few constructs from the social psychological literature have been applied to ADHD research, which seems ill-informed given the chronic interpersonal maladjustment that tends to co-occur. Accordingly, it would be worthwhile to examine how other mediators of social behavior, such as attachment, personality, selfappraisal, ability to delay gratification, and style of love, affect the outcomes of those with ADHD. Finally, it would be productive to revisit these and similar hypotheses in independent samples-particularly female, nonheterosexual, and noncollege populations-to determine the extent to which these findings generalize.

\section{NOTES}

1. Only males were included in this study to maintain consistency (as a follow-up study) and due to limited available resources to conduct this research. This was considered a reasonable compromise given that a larger segment of the ADHD population is male versus female.

2. The dating milestones composite score is an index of both breadth and timing of dating experience, with lower scores indicating more and earlier experience $($ range $=2.56-18.00)$. Calculation $=(1 / \#$ of dating milestones reached)* average age dating milestones were reached.

3. The sexual milestones composite score is an index akin to the dating milestones composite, but relating to sexual behaviors (range $=$ $1.42-8.50)$. Calculation $=(1 / \# \text { of sexual milestones reached })^{*}$ average age sexual milestones were reached.

4. Because direction and magnitude of follow-up $t$ and Mann Whitney tests are practically evident from group mean scores for diagnostic variables (see Table 1), statistical data are not presented for brevity's sake.

5. For descriptive ease, we are defining positive as meaning more active, earlier, or greater breadth of romantic experience; we acknowledge that such experience that is either indiscriminant or at too early an age can be associated with negative outcomes. 


\section{REFERENCES}

American Psychiatric Association. (1994). Diagnostic and statistical manual of mental disorders (4th ed.). Washington, DC: Author.

Ayduk, O., Mendoza-Denton, R., Mischel,W., \& Downey, G. (2000). Regulating the interpersonal self: Strategic self-regulation for coping with rejection sensitivity. Journal of Personality and Social Psychology, 79, 776-792.

Barkley, R. A., \& Biederman, J. (1997). Toward a broader definition of the age-of-onset criterion for attention-deficit hyperactivity disorder. Journal of the American Academy of Child and Adolescent Psychiatry, 36, 1204-1210.

Barkley, R. A., \& Murphy, K. R. (1998). Attention-deficit hyperactivity disorder: A clinical workbook (2nd ed.). New York: Guilford.

Beck, A. T. (1967). Depression: Clinical, experimental and theoretical aspects. New York: Harper \& Row.

Beck, A. T., \& Beck, R. W. (1972). Screening depressed patients in family practice: A rapid technique. Postgraduate Medicine, 52, 81-85.

Beck, A. T., \& Steer, R. A. (1990). Manual for the Revised Beck Anxiety Inventory. San Antonio, TX: Psychological Corporation.

Biederman, J., Faraone, S., \& Mick, E. (2000). Age dependent decline of ADHD symptoms revisited: Impact of remission definition and symptom subtype. American Journal of Psychiatry, 157, 816-817.

Canu, W. H., \& Carlson, C. L. (2003). Differences in heterosocial behavior and outcomes of ADHD-symptomatic subtypes in a college sample. Journal of Attention Disorders, 6, 123-133.

Clarke, L., Ungerer, J., Chahoud, K., Johnson, S., \& Stiefel, I. (2002). Attention deficit hyperactivity disorder is associated with attachment insecurity. Clinical Child Psychology and Psychiatry, 7, 179-198.

Cohen, J. (1992). A power primer. Psychological Bulletin, 112, 155-159. Conners, C. K. (1973). Rating scales for use in drug studies with children. Psychopharmacology Bulletin, 9, 24-85.

Conners, C. K., Erhardt, D., \& Sparrow, E. (1999). Conners Rating Scales: Revised technical manual. North Tanawanda, NY: MultiHealth Systems.

Corcoran, K., \& Fischer, J. (1987). Measures for clinical practice: $A$ sourcebook. New York: Free Press. 
De Quiros, G. B., \& Kinsbourne, M. (2001). Adult ADHD: Analysis of self-ratings on a behavior questionnaire. Annals of the New York Academy of Sciences, 931, 140-147.

Diener, M. B., \& Milich, R. (1997). Effects of positive feedback on the social interactions of boys with attention deficit hyperactivity disorder: A test of the self-protective hypothesis. Journal of Clinical Child Psychology, 26, 256-265.

Downey, G., \& Feldman, S. I. (1996). Implications of rejection sensitivity for intimate relationships. Journal of Personality and Social Psychology, 70, 1327-1343.

Downey, G., Feldman, S., \& Ayduk, O. (2000). Rejection sensitivity and male violence in romantic relationships. Personal Relationships, 7, 45-61.

Downey, G., Freitas, A. L., Michaelis, B., \& Khouri, H. (1998). The self-fulfilling prophecy in close relationships: Rejection sensitivity and rejection by romantic partners. Journal of Personality and Social Psychology, 75, 545-560.

Downey, G., Khouri, H., \& Feldman, S. I. (1997). Early interpersonal trauma and later adjustment: The mediational role of rejection sensitivity. In D. Cicchetti \& S. L. Toth (Eds.), Developmental perspectives on trauma: Theory, research, and intervention (pp. 85-114). Rochester, NY: University of Rochester Press.

Edwards, G., Barkley, R. A., Laneri, M., Fletcher, K., \& Metevia, L. (2001). Parent-adolescent conflict in teenagers with ADHD and ODD. Journal of Abnormal Child Psychology, 29, 557-572.

Erhardt, D., \& Hinshaw, S. P. (1994). Initial sociometric impressions of attention-deficit hyperactivity disorder and comparison boys: Predictions from social behaviors and from nonbehavioral variables. Journal of Consulting and Clinical Psychology, 62, 833-842.

Feldman, S. S., Rosenthal, D. R., Brown, N. L., \& Canning, R. D. (1995). Predicting sexual experience in adolescent boys from peer rejection and acceptance during childhood. Journal of Research on Adolescence, 5, 387-411.

Gates, G. J., \& Sonestein, F. L. (2000). Heterosexual genital sexual activity among adolescent males: 1988 and 1995. Family Planning Perspectives, 32, 295-297, 304.

Gaub, M., \& Carlson, C. L. (1997). Behavioral characteristics of DSM-IV ADHD subtypes in a school-based population. Journal of Abnormal Child Psychology, 25, 103-111. 
Gomez, R., \& Gomez, A. (2002). The effects of perceived maternal parenting styles on the disruptive behaviors of children with attention deficit hyperactivity disorder/oppositional defiant disorder: Mediation by hostile biased social cognitions. In S. P. Shohov (Ed.), Advances in psychology research (pp. 37-55). Hauppauge, NY: Nova Science Publishers.

Hansen, C., Weiss, D., \& Last, C. G. (1999). ADHD boys in young adulthood: Psychosocial adjustment. Journal of the American Academy of Child and Adolescent Psychiatry, 38, 165-171.

Henker, B., \& Whalen, C. K. (1999). The child with attention-deficit/ hyperactivity disorder in school and peer settings. In $\mathrm{H}$. $\mathrm{C}$.

Quay \& A. E. Hogan (Eds.), Handbook of disruptive behavior disorders (pp. 157-178). New York: Plenum.

Hinshaw, S. P., \& Melnick, S. M. (1995). Peer relationships in boys with attention-deficit hyperactivity disorder with and without comorbid aggression. Development and Psychopathology, 7, 627-647.

Hoza, B., Gerdes, A. C., Mrug, S., Hinshaw, S. P., Bukowski, W. M., Gold, J. A., et al. (2005). Peer-assessed outcomes in the Multimodal Treatment Study of children with attention deficit hyperactivity disorder. Journal of Clinical Child and Adolescent Psychology, 34, 74-86.

Hoza, B., Pelham, W. E., Dobbs, J., Owens, J. S., \& Pillow, D. R. (2002). Do boys with attention-deficit hyperactivity disorder have positive illusory self-concepts? Journal of Abnormal Psychology, 111, 268-278.

Hoza, B., Waschbusch, D. A., Pelham, W. E., Molina, B.S.G., \& Milich, R. (2000). Attention-deficit/hyperactivity disordered and control boys' responses to social success and failure. Child Development, 71, 432-446.

Johnston, C., Murray, C., Hinshaw, S. P., Pelham, W. E., \& Hoza, B. (2002). Responsiveness in interactions of mothers and sons with ADHD: Relations to maternal and child characteristics. Journal of Abnormal Child Psychology, 30, 77-88.

Kaufman, A. S. (1990). Assessing adolescent and adult intelligence. Boston: Allyn \& Bacon.

Landau, S., \& Moore, L. A. (1991). Social skills deficits in children with attention-deficit hyperactivity disorder. Reading \& Writing Quarterly, 14, 83-105. 
Locke, H. J., \& Wallace, K. M. (1959). Short marital-adjustment and prediction tests: Their reliability and validity. Marriage and Family Living, 21, 251-255.

Maedgen, J. W., \& Carlson, C. L. (2000). Social functioning and emotional regulation in the attention deficit hyperactivity disorder subtypes. Journal of Clinical Child Psychology, 29, 30-42.

Milich, R., \& Landau, S. (1982). Socialization and peer relations in hyperactive children. In K. D. Gadow \& I. Bialer (Eds.), Advances in learning and behavioral disabilities (Vol. 1, pp. 283-339). Greenwich, CT: JAI Press.

Milich, R., \& Okazaki, M. (1991). An examination of learned helplessness among attention-deficit hyperactivity disordered boys. Journal of Abnormal Child Psychology, 19, 607-623.

Mrug, S., Hoza, B., \& Gerdes, A. C. (2001). Children with attention-deficit/ hyperactivity disorder: Peer relationships and peer-oriented interventions. In D. W. Nangle \& C. A. Erdley (Eds.), The role of friendship in psychological adjustment (pp. 51-77). San Francisco: Jossey-Bass.

Murphy, K., \& Barkley, R. A. (1996). Attention deficit hyperactivity disordered adults: Comorbidities and adaptive impairments. Comprehensive Psychiatry, 37, 393-401.

Murphy, P., \& Schachar, R. (2001). Use of self-ratings in the assessment of symptoms of attention deficit hyperactivity disorder in adults. American Journal of Psychiatry, 157, 1156-1159.

Ohan, J. L., \& Johnston, C. (2002). Are the performance overestimates given by boys with ADHD self-protective? Journal of Clinical Child Psychology, 31, 230-241.

Pelham, W. E., \& Bender, M. E. (1982). Peer relationships in hyperactive children: Description and treatment. In K. D. Gadow \& I. Bialer (Eds.), Advances in learning and behavioral disabilities (Vol. 1, pp. 365-436). Greenwich, CT: JAI Press.

Pliszka, S. R., Carlson, C. L., \& Swanson, J. M. (1999). ADHD with comorbid disorders: Clinical assessment and management. New York: Guilford.

Purdie,V., \& Downey, G. (2000). Rejection sensitivity and adolescent girls' vulnerability to relationship-centered difficulties. Child Maltreatment, 5, 338-349.

Robin, A. L., \& Payson, E. (2002). The impact of ADHD on marriage. The ADHD Report, 10, 9-11, 14. 
Rosenberg, M. (1979). Conceiving the self. New York: Basic Books. Shaw-Zirt, B., Popali-Lehane, L., Chaplin,W., \& Bergman, A. (2005). Adjustment, social skills, and self-esteem in college students with symptoms of ADHD. Journal of Attention Disorders, 8, 109-120.

Stevens, G., \& Featherman, D. L. (1981). A revised sociometric index of occupational status. Social Science Research, 10, 364-395.

Turner, R. J., Frankel, B. G., \& Levin, D. M. (1983). Social support: Conceptualization, measurement, and implications for mental health. Research in Community and Mental Health, 3, 67-111.

Ward, M. F., Wender, P. H., \& Reimherr, F. W. (1993). The Wender Utah Rating Scale: An aid in the retrospective diagnosis of childhood attention deficit hyperactivity disorder. American Journal of Psychiatry, 150, 885-890.

Wechsler, D. (1981). Wechsler Adult Intelligence Scale_Revised (WAIS-R). New York: Psychological Corporation.

Weiss, G., \& Hechtman, L. T. (1993). Hyperactive children grown up. New York: Guilford.

Weiss, G., Hechtman, L., Milroy, T., \& Perlman, T. (1985).

Psychiatric status of hyperactives as adults: A controlled prospective 15-year follow-up of 63 hyperactive children. Journal of the American Academy of Child Psychiatry, 24, 211-220.

Weiss, G., Hechtman, L., Perlman, T., Hopkins, J., \& Wener, A. (1979). Hyperactives as young adults. Archives of General Psychiatry, 36, 675-681.

Weiss, M., Hechtman, L. T., \& Weiss, G. (1999). ADHD in adulthood: A guide to current theory, diagnosis, and treatment. Baltimore, MD: Johns Hopkins University Press.

Wilens, T. E., Biederman, J., \& Spencer, T. J. (2002). Attention-deficit/ hyperactivity disorder across the lifespan. Annual Medical Review, 53, 113-131. 